\title{
Design of the Network Platform Scheme Based on Comprehensive Information Sharing of Zigong City's Characteristic Agriculture
}

\author{
Wen Lei, Hong Zhang, and Lecai Cai
}

School of Computer Science, Sichuan University of Science \& Engineering, Zigong, P.R. China

suselwen@126. com

\begin{abstract}
A network platform scheme targets the Zigong City's characteristic agriculture is designed, which is according to the actuality of characteristic agriculture, the requirements of Comprehensive Information sharing, and the status of city's network topology. In the scheme, many solutions are given out, such as the network architecture, distributed data storage, remote diagnosing, expert decision-making, comprehensive information sharing, distance learning \& training, information managing, and the single sign-on logging, etc. Finally, the capability and security of the network scheme is analysed and summarized.
\end{abstract}

Keywords: Agriculture, Comprehensive information sharing, Network platform, distance learning \& training, Network architecture.

\section{Introduction}

Agricultural informatization is the foundation and important way for modern agricultural development, it can reduce the investment, improve the quality and quantity of the agricultural production, reduce the influence of natural disasters, accelerate agricultural circulation, guide agricultural production and consumption. In market environment, it can help to optimize agricultural resources allocation, reduce market risk, accelerate the spread and popularization of agricultural technology, promote agricultural sci-tech personnel training, and improve the international competitiveness of agricultural products. The network is an important way to realize the agricultural informatization. This paper will aim at Zigong's characteristic agriculture needs to build a comprehensive information sharing network platform.

The platform is an agricultural comprehensive information sharing platform, which includes many functions, such as crop condition monitoring, remote diagnosing, expert decision, comprehensive information sharing, information release and management, products display, market information and instant communication function, etc. The platform is an important tool for users to store, look up and share information, which can collect the internal and external information, then classify and store them in database server. Through the platform, the merchants and agricultural leading department and farmers can share the information. The platform can help merchants and households easily to get market and product information, assist agricultural experts and agricultural leading department to do real-time guides for agricultural production. 


\section{Zigong Characteristic Agriculture Status}

Zigong city locates in the South of Sichuan Basin, is a moist monsoon climate featuring subtropical zone. Its climate is beneficial to develop agriculture. According to the characteristics of Zigong, the city government has mapped out a regional planning of Zigong characteristic agriculture. The characteristic agricultural production is relatively concentrated, and gradually formed a certain scale of industrialized production pattern. At present, the main characteristic agriculture has more than 10 kinds, with the regional distribution in the area two district and four counties. But, in the characteristic agricultural production, there are still some problems, such as follows: (1)The quantity of agricultural sci-tech personnel is less, and most of them live in city, once there are some technological difficulties in production, farmers could hardly obtain the solution timely; (2)In a broad area, farmers are scattered here and there, it is difficult to concentrate to popularize agricultural scientific and technological knowledge, in addition, the agricultural leading department is also hard to grasp agricultural production status, and provide technological guidance;(3)It is not sufficient and timely to take the products market information, farmers are hardly to according the market demand to obtain the maximum economical efficiency;(4)The sales channel is single. Currently, the main sales mechanisms is merchants according to their own demands to buy produce from farmer, on the contrary, due to the lack of market information, farmers can hardly sale their produce to merchants actively, which is easily to cause the product backlog.

To solve the above problems, it is the effective means to build a comprehensive information sharing platform. Through the platform, the farmers can easily gain agricultural science and technological information and market information, the agricultural leading department can also easily grasp the agricultural production status and guide agricultural production.

\section{The Overall Design of Sharing Platform Scheme}

The information sharing platform is a distributed system structure. The planting and breeding belt establish their own regional information center, with Web integration technology, regional information is integrated to the sharing platform of city agricultural information center. The sharing platform through the city telecom network communicates with the regional information center.

\subsection{The Design of Network Topology}

The platform network is constructed to a distributed network structure, the city agricultural information center LAN links to the regional information center network by city telecom network. Remote users can access the sharing platform through Internet, and local users through the city telecom network access the sharing platform or corresponding regional information center. The network topology is shown in figure 1. 


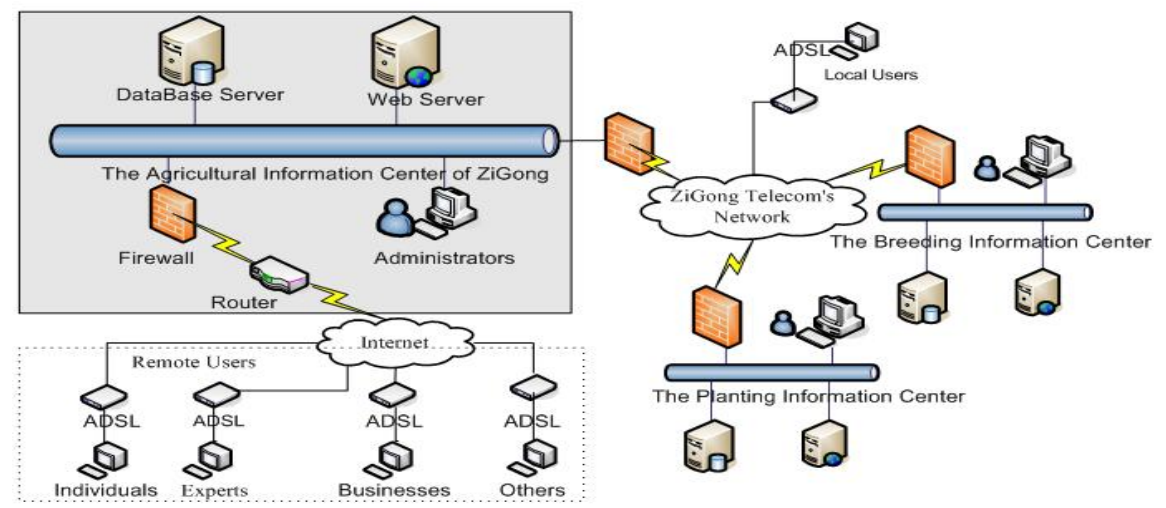

Fig. 1. The Network Structure

\subsection{The Design of Sharing Platform Scheme}

According to the city characteristics agriculture information sharing demand, the platform construction tasks can be divided into 5 subsystems, includes expert system, the remote diagnosis system, information collection and release system, remote learning and training system, and single sign-on(SSO) system. The sharing platform structure is shown in figure 2. Among them, the front 4 systems will be independently developed, this scheme focuses on the single sign-on system.

\section{The Detailed Design Scheme}

In practical applications, most farmers have low level of computer skill, they can only do simple network operation, and most time, the characteristic agricultural planting and breeding farmers are concerned with the agricultural science and technology and the basic pest control information, therefore, the regional information center is the main visit position. According to the practical needs, in sharing platform design, the characteristic agriculture basic information platform and comprehensive information sharing platform are combined the scheme. Each regional information center constructs its basic information platform, and in agricultural science and technology center set up a comprehensive information sharing platform, through the Web integration technology, regional information are integrated to the sharing information platform. Regional information center establish their own expert system, the basic information collection and release system, crop condition monitoring system and basic agriculture science and technology consultation system. The remote diagnosis, system information collection and release system, remote learning and training system and single sign-on system are established in the sharing platform. 


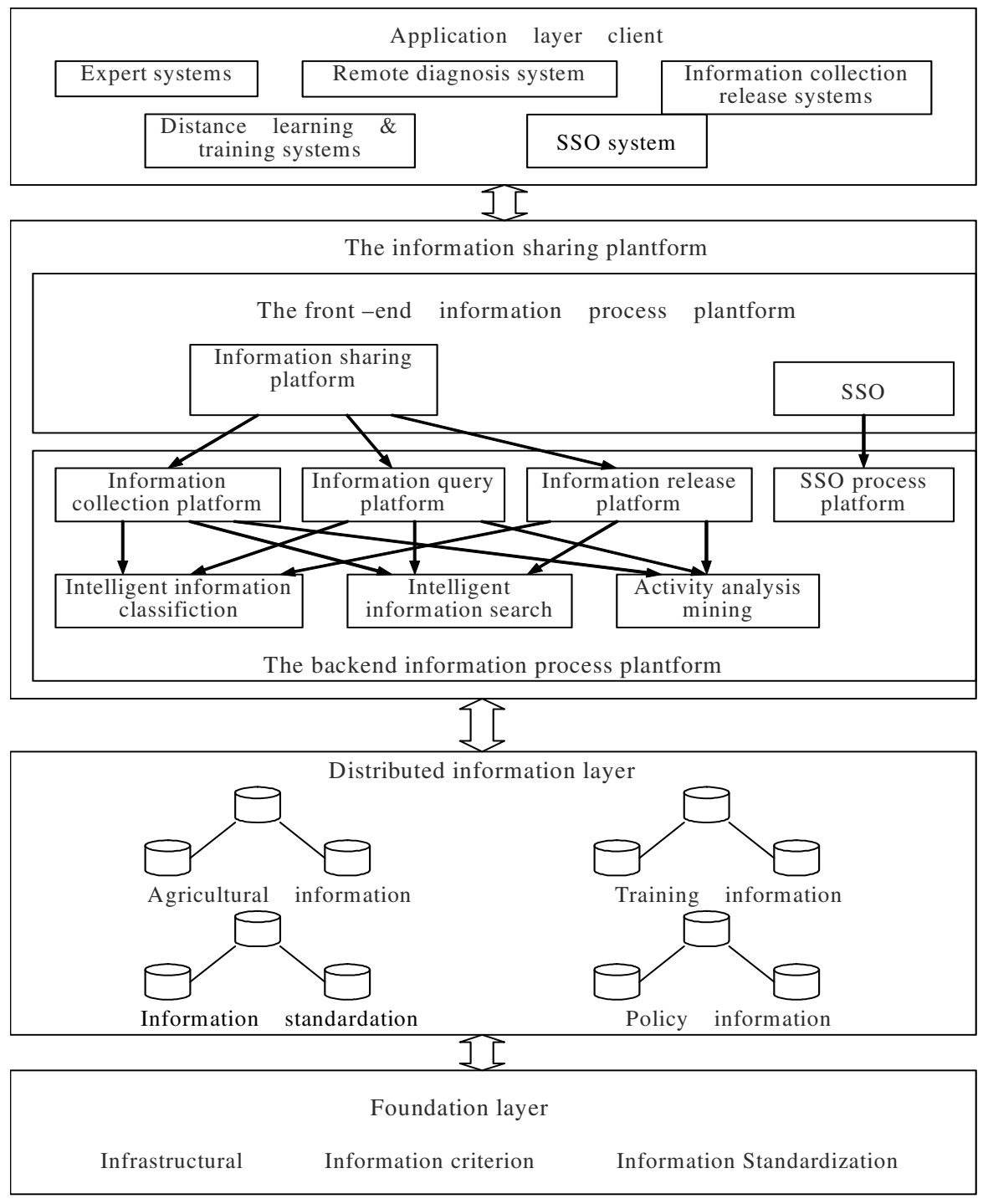

Fig. 2. The structure chart of information sharing platform

\subsection{Single Sign-On System}

Single sign-on(SSO) system is mainly for the convenience of sharing platform and the regional information center management, the system architecture is shown in figure 3 .

Each sharing platform administrator, agricultural science and technology personnel or expert perhaps needs to maintain multiple applications, through the SSO system, he can only need once login to do all of information modification, maintenance, and management of sharing platform and corresponding regional information center. 


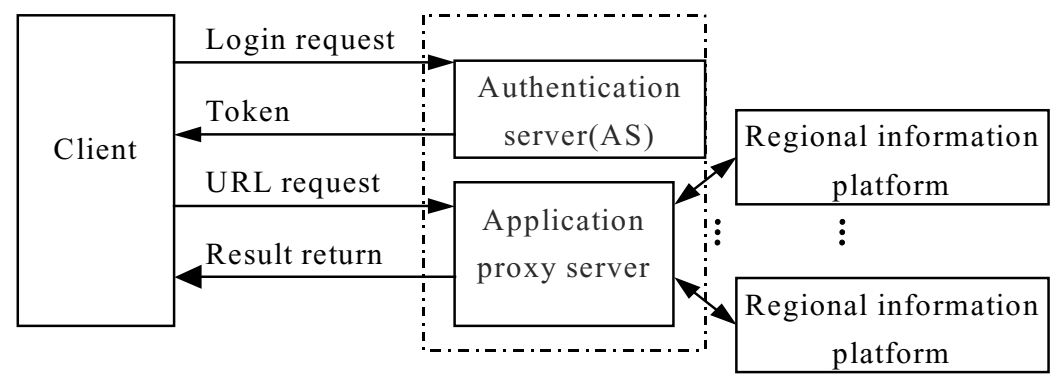

Fig. 3. SSO system architecture

The system is based on Web Service Structure. Each regional basic information platform must register its restricted access application system and set an application proxy in single sign-on system. The proxy contains a registration information table with the URL of the registration application system, replaces user to communicate with the application. The proxy and the basic information platform share a key for secure communications. Each user who needs to access restricted access application must register in single sign-on system, except the identity information, also he must register the information of applications that he can access. After loginning successful, the system distributes the authorization to user by applying for registration information. The authorization is a token form. Once user obtains the authorization, in token validity, he can access the corresponding application, without needing to relogin.

\subsubsection{The Design of Users' Data Structures}

As the uers' information database is used to store many important information, such as the user accounts and passwords, etc, it is vulnerable to attack. In designing, 3 key data tables are used to ensure the database's security, such as user basic information table(UserInfo), user token information table(Token) and user agent data table(URLAgent), they are shown in table 1, table 2 and table 3.

Table 1. UserInfo

\begin{tabular}{lll}
\hline Field names & Description & Remarks \\
\hline User_ID & the unique user ID & Primary key \\
User_name & User name & \\
User_pw & Password & Encypted by MD5 \\
User_Role & User role & Foreign key \\
\hline
\end{tabular}

Table 2. Token

\begin{tabular}{lll}
\hline Field names & Description & Remarks \\
\hline SessID & Token ID & Primary key \\
UserID & User name & Foreign key \\
CurrentIP & User IP address & \\
ExpireTime & Termination time of user session & \\
\hline
\end{tabular}


Table 3. URLAgent

\begin{tabular}{lll}
\hline Field names & Description & Remarks \\
\hline URLAgentID & URLAgent ID & Primary key \\
User_ID & the unique identifier of user & Foreign key \\
URL & The acquisition URL & Encypted by DES \\
POST & HTTPPOST data & Encypted by DES \\
HEADER & HTTP header data & Encypted by DES \\
REFER & ReferURL & Encypted by DES \\
DESURL & Destination URL & Encypted by DES \\
\hline
\end{tabular}

\subsubsection{The Design of Login Certification Process}

The client is realized by plug-in, IE browser and Windows resource management are supported. It uses the Web Service SOAP protocol to interact with authentication server(AS). User login certification process is as follows:

1)The client enables HTTPS to connect the AS, and then sends the user name and password to AS by SOAP.

2) After receiving the information, the AS computes the password with MD5, then matches the result with the corresponding encrypted information in database. If they are mismatching, the AS refuses the login request, else, the AS sends an authentication token and URL field data(called URLCache List) to the client. The URLCache List is extracted from URLAgent table according to User_ID.

3) The AS inserts a new record into Token table. The record consists of SessID, UserID, CurrentIP and ExpireTime.

Through the above steps, the user logins successfully. Then through the URL of URLCache List, he can access the corresponding service directly. In token validity, he does not need to relogin.

\subsection{The Independently Developing System}

\subsubsection{Expert System}

Expert system is an intelligent information system with characteristic agriculture domain expert-level knowledge and experience. Since most farmers are lack of computer application skill, the system adopts question and mouse-click means to interact with users, according to mutual information, it can carry out the corresponding intelligent decision-making for users. In daily agricultural production, once insect appears, farmers can easily use the expert system to obtain the corresponding solved methods and skills.

\subsubsection{Remote Diagnosis System}

If the user cannot obtain satisfactory result by expert system, they can try the remote interactive diagnostic with technical personnel or agricultural experts through the remote diagnosis system. The system is used video transmission to implement, which has 
2 ways of off-line diagnosis and online diagnosis. For off-line diagnosis, users can use photograph, text, pictures and graphics to collect information, and send the messages to the remote diagnostic system. After receiving the messages, system classifies them and submits them to the corresponding technical personnel or expert. When expert offers the solution, system will answer back to the user. For online diagnosis, using video interaction method to implement instant communication, users can interact with experts by using voice, text, video, images and graphics.

\subsubsection{System Information Collection and Release System}

Information collection and release system is divided into 2 parts, the regional information center information collection and release system and the sharing platform of information collection and release system.

The regional information center information collection and release system is mainly used to collect the information of crop growth, environment and agricultural products by artificial method or acquisition terminal, and the regional central database gathers, analysis, processing and issuing the information. Users can monitor crops state through the regional central Web.

The sharing platform information collection and release system mainly includes 2 functions. One is using "link" search technology to gather products information from the regional information center, then classify and release them to the characteristic agriculture exhibition hall of sharing platform. The other is using resources location information retrieval technology and Web mining technology to collect the information of product and market demand, then classifying, analyzing, processing and release, and realize the subscription to users.

\subsubsection{Distance Learning and Training System}

Distance learning and training system offers video courseware and real-time teaching services for users. Usually, the user can use video courseware to learn agricultural technology intuitively. If there is any technical training, remote training can be held through the real-time teaching system. Real-time teaching system is a multicast real-time teaching system, which is adopted with MPEG4 standard and video compression technology of H.264 standard, based on T.120 standards to develop and implement. It can also withstand over 500 user terminals online class at the same time, and provide many functions, such as the audio and video interacting, whiteboard sharing, document sharing, collaborative browsing etc.

\section{Scheme Performance Analysis}

The scheme has higher communication performance and security performance of network communication.

\subsection{Network Performance}

The sharing platform is based on city telecom broadband network, which is a distributed network system structure. As most access businesses occur in regional information 
center, the traffic is effectively reduced on the information sharing platform, it does not cause an access bottleneck in sharing platform.

\subsection{Security Performance}

On the network boundary, firewall is configured, which can be effectively against the denial of service attacks. The access control by security certification can be effectively defense unauthorized access. Between the single sign-on system and regional information center, shared key is used to implement symmetric encryption communication, which can ensure confidentiality of information.

\section{Conclusion}

Aiming at Zigong characteristic agriculture production and marketing demand, this comprehensive information sharing platform is designed, which is planned to complete within 3 years. So far, the constructions of the city information center agricultural information network and the basic information platform of the black goat breeding center of construction have completed. With this scheme achievement and application, it will strongly promote the city characteristic agricultural development with intensification, scale and commercialization, improve agricultural production efficiency and benefit, and speed up the agricultural informatization construction.

\section{References}

1. Ye, L., Luo, M., Chen, J.-h.: Construction of Agriculture Information Service System for Midland Amountainous Area. J. Computer and Modernization 171(11), 169-171 (2009)

2. Single sign-on, http://en.wikipedia.org/wiki/Single_sign-on

3. Yang, Z., Chen, X.-y., Zhang, B.: A single sign-on scheme supporting double authentication method. J. Computer Applications 27(3), 595-596 (2007)

4. Wu, Q.: Design of Grid Agricultural Information Service System. J. Hubei Agricultural Sciences 48(8), 1998-2000 (2009)

5. Hu, C.-x.: The Effects of agro-informationon Building Socialist New Countryside and Development Strategy. J. Commercial Research 367(11), 131-134 (2007)

6. Liu, X.-h., Zhang, Z.: Experience and Countermeasures of Promoting Regional Agricultural Economy Development by Agricultural Informationization Construction. J. Agriculture Network Information 11, 48-50, 60 (2009)

7. Chen, P., Diao, H.-j., Zhu, F.: A Wed Based System of Single Sign-On. J. Computer Applications and Software 24(11), 147-149 (2007) 